\title{
Dimensional Analysis
} in

The Identification of

Mathematical Models 
This page is intentionally left blank 


\title{
Dimensional Analysis
}
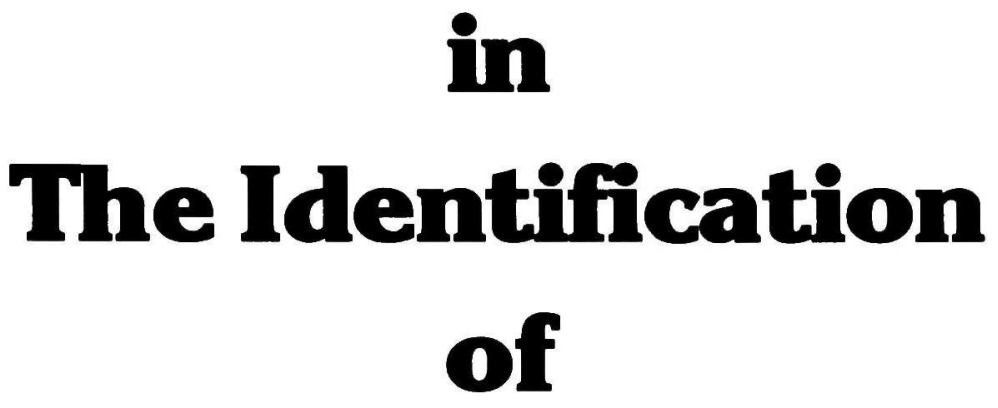

Mathematical Models

\author{
Wacław Kasprzak \\ Bertold Lysik \\ Marek Rybaczuk
}

Technical University of Wroctaw

Poland 


\section{Published by}

World Scientific Publishing Co. Pte. Ltd.

P O Box 128, Farrer Road, Singapore 9128

USA office: 687 Hartwell Street, Teaneck, NJ 07666

UK office: 73 Lynton Mead, Totteridge, London N20 8DH

\section{DIMENSIONAL ANALYSIS IN THE IDENTIFICATION OF MATHEMATICAL MODELS}

Copyright @ 1990 by World Scientific Publishing Co. Pte. Ltd.

All rights reserved. This book, or parts thereof, may not be reproduced in any form or by any means, electronic or mechanical, including photocopying, recording or any information storage and retrieval system now known or to be invented, without written permission from the Publisher.

ISBN 981-02-0304-7

981-02-0305-5 (pbk)

Printed in Singapore by JBW Printers \& Binders Pte. Ltd. 


\section{Preface}

The description of technical, physical and economic processes requires, on the basis of empirical data, the utilization of algorithmic procedures developed within many branches of sciences. They include theories of identification, planning of experiments and dimensional analysis. The theory of systems is the most comprehensive attempt at a synthesis of these theories that have been independently developed. The dimensional analysis, relating to studies of mathematical models on the basis of a priori assumptions of their properties and also the properties of observables, remained on the margin and practically even outside the sphere of interest of specialists engaged in the theory of systems. This work is the result of attempts at constructing algorithmic procedures and programming systems for experiment designing and its data processing. Such systems cannot disregard generally utilized requirements involving mathematical modeling in physics and technology, i.e., invariance concerning defined rotation, translation or gauge groups and also the tensor homogeneity of a model.

These problems have been investigated independently by dimensional analysis [9] and the theory of invariants [35] In utilized mathematical procedures this would require the construction of algorithms in various languages (various spaces). This inconvenience has been removed by the construction of dimensional spaces, based on the theory of fiber bundles, proposed in this work. The authors wish to present two partly independent tasks:

- the use of dimensional analysis in the identification of mathematical models, - a new concept of dimensional analysis which, in their opinion, solves problems hitherto insoluble by the classical method.

For that reason, possibilities of dimensional analysis in identification tasks and its influence on possible strategies in solving this problem, are discussed in Chapters one to three, on the basis of the already classical version. This provided an opportunity to reveal all its shortcomings. Chapter four discusses experimenter's proceedings in the search for an empirical model on the base of dimensional analysis. It shows that from the point of view of intellectual tasks faced by an experimenter it is essential to have the possibility:

- of testing the hypothesis concerning the completeness of the set of arguments of a model,

- seeking the lacking arguments,

- and seeking the form of the function $\mathrm{f}$.

For this reason some possibilities of creating experts systems for solving this problem are discussed. There are subsidiary discourses on a new construction of dimensional space (Appendix A), invariant properties of dimensional functions (Ap- 
pendix B) and an identification of invariant functions (Appendix C). The arrangement of the contents was based on the assumption that specialists concerned with the theory of systems may not necessarily be interested in dimensional analysis but rather in its use in the identification process. However, those who are interested in dimensional analysis may look it up in the appendices. Practically, only two problems directly linked to dimensional analysis have been left out there: the selection of a dimensional base (Chapter 2), an issue nonetheless illustrated by an example in Appendix B (cf. example B.8.1) and the rules of correspondence (cf. section 3.1).

We want to express here sincere thanks to $\mathrm{mr}$ Jan Rudzki for english translation of the book.

Authors 


\section{Contents}

1 Drobot's Dimensional Space and a Classical Theory of Measurement

1.1 Drobot's dimensional space . . . . . . . . . . . . . . . . 1

1.2 Problems of the Classical Theory of Measurement . . . . . . . . . 5

2 A Dimensional Analysis and the Construction of Empirical Models

2.1 The dimensional function and Theorem $\pi$ according to Drobot . . . 12

2.2 Theorem $\pi$, and an identification of a mathematical model . . . . 16

2.3 Similarity relation, qualitative information about a mathematical model 20

2.4 The selection of a dimensional base at the approximation of results

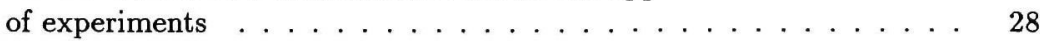

3 Multi-Stage Identification and Dimensional Complex Function

3.1 Multi-Stage Identification, Rule of Correspondence . . . . . . . . . 35

3.2 Complex Dimensional Function, Dimensional Interpretation of Scalar and Vector Fields . . . . . . . . . . . . . . . . . 42

4 Algorithmic Procedures in the Construction of Empirical Models. Conclusions

4.1 An Analysis of an Experimenter's Proceedings in the Search for an Empirical Model . . . . . . . . . . . . . . . . . . . . . . 48

4.2 Testing the Hypothesis Concerning the Completeness of the Set of Arguments of Function (2.1) and Searching for Lacking Arguments . 51

4.3 Seeking the Form of Function $f \ldots \ldots \ldots \ldots$

A Dimensional Spaces - Description of the Construction

A.1 Groups Action on Sets . . . . . . . . . . . . . . . 61 61

A.2 Drobot's Dimensional Spaces . . . . . . . . . . . . . . . 63

A.3 Generalized Dimensional Spaces . . . . . . . . . . . . . 69

A.4 Algebra of Dimensional Quantities. Interpretations. . . . . . . . 73

A.5 Gauge Group $\operatorname{Hom}\left(\mathcal{W}, \mathbf{R}_{+}\right) \ldots \ldots \ldots \ldots$

A.6 Abstract Dimensional Spaces . . . . . . . . . . . . . . . . 83

A.7 Dimensional Symmetry and Geometry . . . . . . . . . . 88

A.8 Dimensional Spaces and the Measurement Theory. Interpretations . . 104 
B Theorem $\pi$ Including the Geometry of Dimensional Quantities

B.1 The Invariant Functions . . . . . . . . . . . . . . . . . . . 109

B.1.1 Examples . . . . . . . . . . . . . . . . . 113

B.2 Theorem $\pi$ for Drobot's Dimensional Space. . . . . . . . . . 116

B.3 The Constructions in Dimensional Geometry . . . . . . . . . . . 121

B.4 Theorem $\Pi$ for the $\mathbb{W W}(R)$ Space . . . . . . . . . . . . . . . . 129

B.5 Theorem $\Pi$ for $\mathbb{W W}\left(R^{3}\right) \ldots \ldots \ldots \ldots \ldots$

B.6 Theorem $\pi$ for the space $\mathbb{I I I}_{\oplus} \ldots \ldots \ldots \ldots \ldots 14 \ldots \ldots \ldots$

B.7 Cases of Special Groups of Symmetry . . . . . . . . . . . . . . 151

B.8 Examples of the Application of Theorem $\pi \ldots \ldots \ldots 152$

B.8.1 Example 1: Bernoulli's Law . . . . . . . . . . . . . 152

B.8.2 Example 2: Stresses in Planar Plates and Discs . . . . . . . 159

B.9 Subgroups of the Rotation Group. Other "Dimensions". . . . . . . 170

C An Identification of Invariant Functions . . . . . . . . . . 178

C.1 Examples . . . . . . . . . . . . . . . . . . . 188

C.1.1 Example 1. . . . . . . . . . . . . . . . . . . 188

C.1.2 Example 2 . . . . . . . . . . . . . . . . . . . 189 